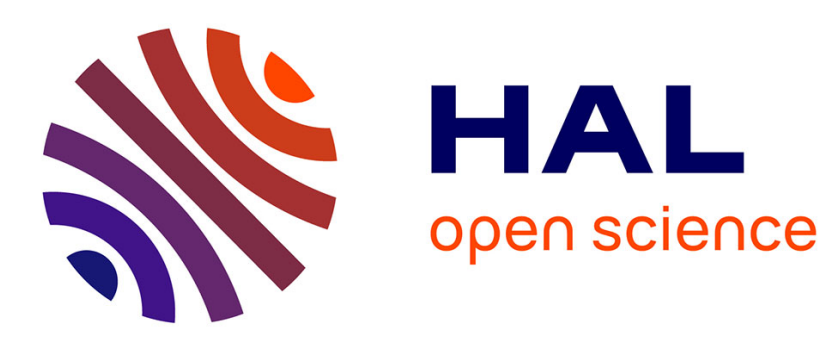

\title{
Espaces scolaires et éducatifs : les liaisons nouvelles entre l'école et le champ éducatif
}

Benjamin Moignard, Thomas Sauvadet

\section{To cite this version:}

Benjamin Moignard, Thomas Sauvadet. Espaces scolaires et éducatifs : les liaisons nouvelles entre l'école et le champ éducatif. Espaces et sociétés (Paris, France), 2016, 166 (3), pp.7-13. 10.3917/esp.166.0007 . hal-01615556

\section{HAL Id: hal-01615556 https://hal.science/hal-01615556}

Submitted on 19 Oct 2017

HAL is a multi-disciplinary open access archive for the deposit and dissemination of scientific research documents, whether they are published or not. The documents may come from teaching and research institutions in France or abroad, or from public or private research centers.
L'archive ouverte pluridisciplinaire HAL, est destinée au dépôt et à la diffusion de documents scientifiques de niveau recherche, publiés ou non, émanant des établissements d'enseignement et de recherche français ou étrangers, des laboratoires publics ou privés. 
CHERCHER, REPÉRER, AVANCER

Éditorial

ESPACES SCOLAIRES ET ÉDUCATIFS : LES LIAISONS NOUVELLES ENTRE L'ÉCOLE ET LE CHAMP ÉDUCATIF

Benjamin Moignard, Thomas Sauvadet

ERES | Espaces et sociétés 》

2016/3 $n^{\circ} 166 \mid$ pages 7 à 13

ISSN 0014-0481

ISBN 9782749251523

Article disponible en ligne à l'adresse :

https://www.cairn.info/revue-espaces-et-societes-2016-3-page-7.htm

\section{Pour citer cet article :}

Benjamin Moignard, Thomas Sauvadet« Espaces scolaires et éducatifs : les liaisons nouvelles entre l'école et le champ éducatif », Espaces et sociétés 2016/3 (n 166), p. 7-13.

DOI 10.3917/esp.166.0007

Distribution électronique Cairn.info pour ERES.

(C) ERES. Tous droits réservés pour tous pays.

La reproduction ou représentation de cet article, notamment par photocopie, n'est autorisée que dans les limites des conditions générales d'utilisation du site ou, le cas échéant, des conditions générales de la licence souscrite par votre établissement. Toute autre reproduction ou représentation, en tout ou partie, sous quelque forme et de quelque manière que ce soit, est interdite sauf accord préalable et écrit de l'éditeur, en dehors des cas prévus par la législation en vigueur en France. Il est précisé que son stockage dans une base de données est également interdit. 
I

ESPACES SCOLAIRES ET ÉDUCATIFS 


\title{
Éditorial Espaces scolaires et éducatifs : les liaisons nouvelles entre l'école et le champ éducatif
}

\author{
Benjamin Moignard \\ Thomas Sauvadet
}

\begin{abstract}
C e numéro s'intéresse à la recomposition des espaces scolaires et éducatifs en rapport avec la prise en charge des problématiques sociales et scolaires qui marquent l'école contemporaine. Les mouvements de massification scolaire constatés dans la plupart des systèmes éducatifs des cinquante dernières années ont considérablement transformé les enjeux liés à l'espace scolaire. Si l'école est confortée dans ses fonctions de socialisation et d'encadrement de la jeunesse, si les niveaux de formation des nouvelles générations sont supérieurs à ceux des précédentes, si les aspirations à la démocratisation scolaire sont partagées et se sont imposées comme un enjeu majeur de développement économique, voire démocratique, aucun système éducatif ne parvient à résoudre correctement l'équation qui permettrait d'assurer l'égalité d'accès et de réussite des élèves, indépendamment de leurs milieux sociaux d'origines. Que ce soit dans les pays occidentaux à partir des années 1980 pour le second degré (Merle, 2002), en Amérique Latine à partir des années 1990 dans le premier puis le second degré (Peregrino, 2010), ou dans de nombreux pays africains et asiatiques (Akkari et Payet, 2010), l'accroissement de la scolarisation ne suffit jamais à conjurer les inégalités sociales de réussite à l'école. Le poids du diplôme est démultiplié dans la logique de distribution des positions
\end{abstract}


sociales (Millet et Moreau, 2011), alors que dans le même temps, les dispositifs d'orientation renforcent des logiques institutionnelles qui s'articulent mal aux formes d'appropriation de l'école par les familles populaires (Chauvel, 2015).

Si la place de l'école dans la construction des inégalités sociales reste d'une redoutable actualité, il y a sans doute un piège à privilégier une approche par trop scolaro-centrée, qui considère l'école pour ce qu'elle devrait être plutôt que pour ce qu'elle est. Ainsi, il est sans doute indispensable d'interroger de manière plus approfondie les mouvements de requalification de la place de l'école dans nos sociétés et l'émergence de nouveaux espaces éducatifs qui témoignent d'une recomposition des formes d'encadrement et de socialisation de la jeunesse. Il y a donc sans doute un intérêt à ce que la sociologie de l'éducation, et c'est peut-être plus vrai en France qu'ailleurs, ne soit pas seulement une sociologie de l'école. Bien sûr, dans une société qui témoigne d'une véritable «passion » en la matière, et alors que l'on connaît le poids de l'expérience scolaire sur les destins sociaux (Dubet, Duru-Bellat et Vérétout, 2010), il reste indispensable d'approfondir et de prolonger des travaux qui ont fait l'histoire de la discipline. Mais comme l'écrit clairement Patrick Rayou dans un ouvrage récent, on observe une mobilité des frontières de l'école qui ne peut plus être considérée comme un espace protégé du monde, car «les sociétés ouvertes suscitent des écoles ouvertes » (Rayou, 2015, p. 10). Ces écoles, moins monolithiques et fermées sur elles-mêmes, n'ont plus le monopole de la diffusion des savoirs et de l'encadrement des jeunes pendant le temps scolaire, et des liaisons nouvelles s'opèrent avec des espaces éducatifs qui intègrent d'autres formes de socialisations éducatives ou socioculturelles : dispositifs à destination de publics spécifiques, accueils périscolaires, espaces d'animation et d'éducation populaire, structures socio-éducatives...

La désignation de "problèmes publics » (Neveu, 2015) associée aux cadres de l'école massifiée et à ses nouveaux publics, interroge l'articulation de ses missions ordinaires autour de l'instruction et de la transmission des apprentissages, avec des formes éducatives plus larges. La plupart des pays démocratiques sont ainsi traversés depuis une trentaine d'années par de fortes préoccupations autour de ces «nouvelles problématiques éducatives 》 que sont devenus l'échec scolaire, la violence à l'école, le décrochage scolaire ou les discriminations de tous ordres dans l'espace scolaire qui, parce qu'ils débordent les cadres traditionnels de la socialisation scolaire et bénéficient de formes de prise en charge localisées, cristallisent la transformation des liaisons entre les espaces scolaires et éducatifs (Moignard et Rubi, à paraître). Ce sont des éducateurs qui s'occupent des élèves exclus (article de Juliette Garnier), des animateurs qui exercent au cœur même de l'école (article de Julien Netter), des parents qui deviennent acteurs de la vie scolaire (article de Genviève Zoïa et Laurent Visier). Ce sont aussi les échelles d'intervention qui se transforment, la mobilisation locale complétant ou suppléant, selon les points de vue, les 
politiques nationales. Cet échelon de la proximité et les structures qui l'administrent (collectivités locales, associations...), deviennent parties prenantes des politiques éducatives, alors que l'État reconfigure en France et ailleurs ses modalités d'intervention en la matière (Buisson-Fenet et Pons, 2014).

À partir de l'exemple du traitement de la mixité sociale à l'école au niveau départemental et régional, la contribution de Choukri Ben Ayed, démontre une forte spatialisation des problèmes éducatifs. Cette spatialisation accentue la propension des acteurs locaux à agir, non pas sur les structures de l'action éducative de proximité (sectorisation, politiques d'établissements scolaires, contrôle des dérogations), mais sur les populations ségréguées, dans une logique de normalisation qui promeut des interventions autour de la citoyenneté, de la parentalité ou de l'interculturalité par exemple.

Cette mise en perspective se prolonge avec la contribution d'Étienne Douat qui, en s'appuyant sur une enquête ethnographique concernant les exclusions de classe dans huit collèges et lycées de secteurs populaires, illustre la difficulté à faire vivre au quotidien la mixité lorsque les disparités sociales et scolaires se déclinent au cœur des espaces scolaires. Il montre ainsi comment le recours à l'exclusion des éléments les plus perturbateurs marque une protection de la skholè et une distribution spatiale des corps, qui légitiment les procédures d'externalisation et de délégation de la prise en charge de ces « problèmes », sur fond de division du travail éducatif qui met en péril selon lui, l'idée même de travail en équipe.

Il est aussi question de mise à l'écart d'élèves dans la contribution de Juliette Garnier qui s'intéresse, dans un cadre extra-scolaire, à la prise en charge des exclus au collège. Elle restitue un exemple de ces nouvelles formes d'organisation scolaire que sont les « dispositifs locaux », perçus comme autant de moyens de répondre à l'hétérogénéité des publics et à des contraintes locales perçues comme spécifiques (Barrère, 2013), là où l'on pourrait voir plutôt la manifestation d'une spatialisation des problèmes sociaux qui homogénéise le traitement des populations les plus précaires. Elle aborde les effets d'une prise en charge qui se heurte à la difficulté de traduire en acte des alliances éducatives qui débordent le seul enjeu de soulager l'école de la présence des élèves désignés comme perturbateurs : comment ne pas céder à une logique d'externalisation de ces prises en charge qui marquerait non pas tant une logique partenariale que l'abandon par l'école de certaines de ses missions?

Cet enjeu du partenariat est au cœur de l'article de Julien Netter qui étudie l'application de la réforme des rythmes scolaires dans sept écoles de quartiers parisiens, et pose la question de l'appropriation par les élèves des activités culturelles favorables aux apprentissages (éducation à l'image, théâtre...). Tout en illustrant cette recomposition physique de l'école en un espace unifié autour de registres scolaires, éducatifs et socioculturels, cette contribution permet de saisir l'hétérogénéité des buts et des pratiques des encadrants, qui rendent 
difficiles, pour les élèves de milieux populaires en particulier, l'appropriation des différents registres d'apprentissages auxquels ils sont confrontés. L'offre culturelle de ces écoles parisiennes, développée par le biais d'intervenants plutôt habitués aux publics favorisés, ne suffit pas à créer les conditions de l'appropriation par les élèves de cette forme d'activité intégrée en milieu scolaire, ni à améliorer leur réussite à l'école.

Cette ouverture de l'école à de nouveaux acteurs et à de nouvelles pratiques est une préoccupation qui traverse également la contribution de Geneviève Zoïa et Laurent Visier. Ils proposent une analyse de la démarche de reconstruction d'une école dans un quartier défavorisé d'une ville française de taille moyenne, qui montre les potentialités et les obstacles rencontrés pour favoriser son ouverture aux associations et aux parents d'élèves. L'aménagement des lieux, les formes de reconnaissance et d'intégration favorables à l'avènement d'une école de la proximité et du «bien-être » que les auteurs appellent de leurs vœux, semblent céder la place à une logique de repli et de sécurisation de l'espace scolaire.

La territorialisation des politiques éducatives et ses effets sont également interrogés par Lucy Bell et Pierre-Yves Bernard qui s'intéressent plus particulièrement au traitement du décrochage scolaire dans des territoires urbains et ruraux. Ainsi, il semble que l'organisation spécifique de l'offre de formation sur ces territoires influence les parcours, le rapport à l'institution ainsi que les motifs de décrochage scolaires. Alors que de nombreuses études se focalisent sur les seuls territoires urbains ségrégués, les auteurs montrent qu'une faible offre scolaire, une perspective d'emplois limitée et une mixité sociale relative contribuent à décourager les élèves en difficultés dans les milieux périurbains.

Ces constats recoupent ceux de Marcelo Burgos dans le dernier article de ce numéro, qui questionne les "effets de lieu» dans la construction des parcours scolaires des élèves d'une importante favela de Rio de Janeiro. Ce décalage international permet un regard sur les effets d'une massification scolaire encore en marche, qui interroge la faiblesse structurelle de l'école publique brésilienne face au secteur privé, et sa difficulté à entrer pleinement dans une démarche scolaire et éducative qui lui permette de dépasser les injonctions à prolonger des politiques de gestion et de contrôle de la pauvreté.

Dans une société concurrentielle où les espaces sont de plus en plus hiérarchisés, où les segmentations à l'intérieur de l'école se renforcent, où les frontières extérieures évoluent, il est sans doute nécessaire de continuer d'interroger les liens entre les ségrégations, les politiques locales et territorialisées, les parcours scolaires et les formes de prises en charge et de mobilisations autour de ces nouvelles problématiques éducatives. Les alliances comme les nouvelles formes de division du travail qui se nouent autour de ces questions, témoignent d'une recomposition des espaces scolaires et éducatifs qui marque sans doute durablement une transformation des cadres et des structures 
d'encadrement de la jeunesse. Elles interrogent également les modalités à partir desquelles ces liaisons nouvelles reconfigurent ou transforment les fonctions d'une école qui participe à la distribution méritocratique des positions sociales, tout en contribuant à la reproduction massive des inégalités sociales. Reste donc à définir les conditions à partir desquelles ces recompositions produiront des ressources et des espaces démocratiques renouvelés, indispensable à l'émancipation de toutes les jeunesses.

\section{RÉFÉRENCES BIBLIOGRAPHIQUES}

AKKARI, A. ; PAYET, J.-P. 2010. Transformations des systèmes éducatifs dans les pays du Sud. Entre globalisation et diversification, Bruxelles, De Boeck Supérieur.

BARRÈre, A. 2013. La montée des dispositifs : un nouvel âge de l'organisation scolaire. Carrefours de l'éducation, $\mathrm{n}^{\circ} 36$ (2), p. 95-116.

Buisson-Fenet, H. ; Pons, X. 2014. School Evaluation Policies and Educating States: Trends in Four European Countries, Genève, Peter Lang.

Chauvel, S. 2015. «Le chemin de l'école », Politix, n 108 (4), p. 53-73.

Dubet, F. ; Duru-Bellat, M. ; Vérétout, A. 2010. Les sociétés et leur école. Emprise du diplôme et cohésion sociale, Paris, Le Le seuil.

Merle, P. 2002. La démocratisation de l'enseignement, Paris, La Découverte.

Millet, M. ; Moreau, G. 2011. La société des diplômes, Paris, La Dispute.

MoIGNARD, B. ; RUBI, S. (à paraître). L'école des dispositifs. Approches internationales autour de la prise en charge des nouvelles problématiques éducatives, Genève, Peter Lang.

Neveu, E. 2015. Sociologie politique des problèmes publics, Paris, Armand Colin.

Peregrino, M. 2010. Trajetórias desiguais: Um estudo sobre os processos de escolarização pública de jovens pobres, Rio de Janeiro, Garamond.

RAYOU, P. 2015. Aux frontières de l'école : institutions, acteurs et objets, Vincennes, Presses universitaires de Vincennes. 